\title{
ANALYTIC EXTENSION BY HAUSDORFF METHODS
}

\author{
BY \\ RALPH PALMER AGNEW
}

1. Introduction. Let $\chi(t)$ be a complex-valued function having bounded variation over $0 \leqq t \leqq 1$, let $\chi(0)=0$, and let $\chi(t)$ have no removable discontinuities. This mass function $\chi(t)$ generates a Hausdorff transformation (Hausdorff [9]) $H(\chi)$

$$
\sigma_{n}=\int_{0}^{1} \sum_{k=0}^{n} C_{n, k} t^{k}(1-t)^{n-k} s_{k} d \chi(t)
$$

by means of which a series $u_{0}+u_{1}+u_{2}+\cdots$ with partial sums $s_{0}, s_{1}, s_{2}, \ldots$ is summable $H(\chi)$ to $\sigma$ if $\sigma_{n} \rightarrow \sigma$ as $n \rightarrow \infty$. The transformation $H(\chi)$ is regular (that is, such that existence of $\lim s_{n}$ implies $\lim \sigma_{n}=\lim s_{n}$ ) if and only if $\chi(1)=1$ and $\chi(t) \rightarrow 0$ as $t \rightarrow 0$.

Among the familiar regular transformations obtained by specializing $\chi(t)$ are the methods $C_{r}(R(r)>0)$ of Cesàro for which $\chi(t)=1-(1-t)^{r}$; the methods $H_{r}(R(r)>0)$ of Hölder for which

$$
\chi(t)=\frac{1}{(r-1) !} \int_{0}^{t}\left(\log \frac{1}{u}\right)^{r-1} d u ;
$$

and the methods $E_{r}(0<r \leqq 1)$ of Euler for which $\chi(t)=0$ or 1 according as $0 \leqq t<r$ or $r \leqq t \leqq 1$.

It is well known that neither Cesàro nor Hölder methods are effective in evaluating power series outside their circles of convergence. Characterization of the region of the complex plane in which a power series $\sum a_{n} z^{n}$ is summable $E_{r}$ was given by Knopp [14] and Rademacher [18] for the cases in which $r$ is of the form $2^{-k}, k=1,2,3, \cdots$. If $z_{0}$ is an interior point of the Borel polygon determined by $\sum a_{n} z^{n}$, then $\sum a_{n} z_{0}^{n}$ is summable $E_{r}$ provided $r$ lies within a sufficiently small interval $0<r<\delta$ to the right of the origin. On the other hand, if $z_{1}$ is a point outside the Borel polygon, then no regular Euler method can evaluate $\sum a_{n} z_{1}^{n}$. Beyond these facts and a few corollaries of them, very little seems to be known about the problem of analytic extension by means of Hausdorff methods of summability. It has been conjectured by Garabedian and Wall [8] that a regular Hausdorff method is ineffective outside the circle of convergence unless $\chi(t)$ is constant over some interval $1-\delta \leqq t \leqq 1$. For references to literature (up to 1927) on analytic extension by various methods, see Hille [10].

Presented to the Society, September 5, 1941; received by the editors July 14, 1941. 
Long ago it became the fashion to test the efficacy of methods of summability by applying them to the geometric series $\sum z^{n}$. We determine, in terms of the generating function $\chi(t)$, the open region of the $z$-plane in which $\sum z^{n}$ is summable by a regular Hausdorff method $H(\chi)$. The result is set forth in the following theorem.

THEOREM 1.1. Let $\chi(t)$ have bounded variation over $0 \leqq t \leqq 1$, let $\chi(0+)$ $=\chi(0)=0$, let $\chi(1)=1$, and let $\chi(t)$ have no removable discontinuities. Let $r$ be the greatest lower bound of all numbers $\rho$ such that $\chi(t)=1$ when $\rho \leqq t \leqq 1$. Then $\sum z^{n}$ is summable $H(\chi)$ to $1 /(1-z)$ at each point $z$ interior to the circle

$$
\left|z-\left(1-\frac{1}{r}\right)\right|=\frac{1}{r}
$$

and $\sum z^{n}$ is non-summable $H(\chi)$ at each point $z$ exterior to the same circle.

Let the number $r$ described in the statement of the theorem be termed the order of the transformation $H(\chi)$, and let the circle be termed the circle of summability. If (as is the case for the Cesàro and Hölder methods) there is no interval $\rho<t \leqq 1$ over which $\chi(t)$ is constant, then the order $r$ of $H(\chi)$ is 1 and the circle of summability is identical with the circle of convergence. If (as is the case for the Euler methods of order $0<r<1$ ) there is an interval $\rho<t \leqq 1$ over which $\chi(t)$ is constant, then the order $r$ of $H(\chi)$ is less than 1 . The order must be positive since $\chi(t) \rightarrow 0$ as $t \rightarrow 0$. Thus the radius $r^{-1}$ of the of the circle of summability is greater than 1 , the circle of summability being tangent to the line $R(z)=1$ at the point $z=1$. Since the center and radius of the circle of summability depend only upon the order of $H(\chi)$, it follows that two Hausdorff methods of equal order have the same circle of summability. In particular, the circle of summability of a Hausdorff method of order $r$ is the same as that of the Euler method $E_{r}$ of equal order $\left({ }^{1}\right)$. Differences in effectiveness of two Hausdorff methods of equal order can appear only for points $z$ on the circle of summability. It is a consequence of Theorem 1.1 that the effectiveness of methods $H(\chi)$ in evaluating $\sum z^{n}$ increases steadily as the order $r$ decreases. Each fixed point $z_{0}$ for which $R z_{0}<1$ lies within the circle of summability of $H(\chi)$ provided the order $r$ of $H(\chi)$ is sufficiently near 0 . Each fixed point $z_{1}$ for which $z_{1} \neq 1$ and $R z_{1} \geqq 1$ lies outside each circle of summability and accordingly $\sum z_{1}^{n}$ is non-summable $H(\chi)$ for each regular $H(\chi)$.

Proof of summability of $\sum z^{n}$ when $z$ lies inside the circle of summability is very simple and is given in $\$ 2$. Proof of non-summability is more complicated; this and related facts are proved in $\$ 3$. In $\$ \S 4$ and 5 , we establish uniform summability of power series over appropriate sets. In $\S \S 6$ and 7 , we

(1) A regular method $H(\chi)$ includes $E_{r}$ if and only if the order of $H(\chi)$ is less than or equal to $r$. See Hille and Tamarkin [11]. For a general discussion of inclusion relations involving regular Hausdorff methods, see Garabedian, Hille, and Wall [7]. 
define and discuss collective Hausdorf summability. In $§ 8$, we improve a known theorem relating Abel and Hausdorff summability.

2. Proof of summability. When $z \neq 1$, the series $\sum z^{n}$ has partial sums

$$
s_{k}=\left(1-z^{k+1}\right) /(1-z), \quad k=0,1,2, \cdots,
$$

and the $H(\chi)$ transform of the series $\sum z^{n}$ is accordingly

$$
\begin{aligned}
\sigma_{n}(z) & =\frac{1}{1-z}-\frac{z}{1-z} \int_{0}^{1} \sum_{k=0}^{n} C_{n, k}(t z)^{k}(1-t)^{n-k} d \chi(t) \\
& =\frac{1}{1-z}-\frac{z}{1-z} f_{n}(z)
\end{aligned}
$$

where

$$
f_{n}(z)=\int_{0}^{1}[1+t(z-1)]^{n} d \chi(t) .
$$

The sequence $f_{n}(z)$ is the $H(\chi)$ transform of the sequence $s_{k} \equiv z^{k}$.

Let $\chi(t)$ be regular and let $z_{0}$ be a fixed point inside the circle of summability; we show that $\sum z_{0}^{n}$ is summable to $1 /\left(1-z_{0}\right)$ by showing that $f_{n}\left(z_{0}\right) \rightarrow 0$ as $n \rightarrow \infty$. Since $z_{0}$ lies inside the circle of summability,

$$
\left|1+r\left(z_{0}-1\right)\right|<1
$$

where $r$ is the order of $H(\chi)$ and accordingly $\chi(t)=1$ when $r<t \leqq 1$. We may assume that $\chi(t)=1$ over $r \leqq t \leqq 1$. Let $\epsilon>0$. Then

$$
\begin{aligned}
\left|f_{n}\left(z_{0}\right)\right| & =\left|\int_{0}^{r}\left[1+t\left(z_{0}-1\right)\right]^{n} d \chi(t)\right| \\
& \leqq \int_{0}^{\delta}\left|1+t\left(z_{0}-1\right)\right|^{n}|d \chi(t)|+\int_{\delta}^{r}\left|1+t\left(z_{0}-1\right)\right|^{n}|d \chi(t)| \\
& \leqq \int_{0}^{\delta}|d \chi(t)|+\int_{\delta}^{r} A^{n}|d \chi(t)|<\epsilon
\end{aligned}
$$

when $n$ is sufficiently great, provided $\delta$ is a positive number so chosen that

$$
\int_{0}^{\delta}|d \chi(t)|<\epsilon / 2
$$

and $A$ is a constant for which

$$
\max _{\delta \leqq t \leqq r}\left|1+t\left(z_{0}-1\right)\right| \leqq A<1 .
$$

Hence $f_{n}\left(z_{0}\right) \rightarrow 0$ and our result follows. In case $F$ is a closed set interior to the circle of summability, it is possible to fix $\delta>0$ such that (2.5) holds and 
then show existence of a constant $A$ such that (2.6) holds for each $z_{0} \in F$; this implies uniform summability over the set $F$.

3 . Proof of non-summability. Assuming that $\chi(t)$ satisfies the hypotheses of Theorem 1.1 and that $z$ is a fixed point outside the circle of summability, we show that $\sum z^{n}$ is not summable $H(\chi)$. For this, it is sufficient to show that the $H(\chi)$ transform $f_{n}(z)$ of the sequence $z^{n}$ is unbounded.

The hypothesis that $z$ lies outside the circle of summability implies that $|z|>1$. Hence there is a number $t_{1}$ such that $0 \leqq t_{1}<1$ and

$$
\begin{aligned}
|1+(z-1) t| & \leqq 1, & & 0 \leqq t \leqq t_{1}, \\
& >1, & & t_{1}<t \leqq 1 .
\end{aligned}
$$

In case $R z \geqq 1, t_{1}$ is 0 ; and in case $R z<1$, we have $0<t_{1}<1$. Again using the hypothesis that $z$ lies outside the circle of summability, we obtain

$$
|1+(z-1) r|>1
$$

where $r$ is the order of $H(\chi)$, and accordingly $r>t_{1}$. Hence our result is a consequence of the following theorem.

THEOREM 3.1. If $|z|>1$ and $\chi(t)$ is a function of bounded variation over $0 \leqq t \leqq 1$ having no removable discontinuities, then a necessary and sufficient condition that the $H(\chi)$ transform

$$
f_{n}(z)=\int_{0}^{1}[1+(z-1) t]^{n} d \chi(t)
$$

of the sequence $s_{k} \equiv z^{k}$ be bounded is that $\chi(t)$ be constant over the interval $t_{1}<t \leqq 1$ over which $|1+(z-1) t|>1\left({ }^{2}\right)$.

Sufficiency is obvious; for if $\chi(t)$ is constant over $t_{1}<t \leqq 1$, then we may assume $\chi(t)$ constant over $t_{1} \leqq t \leqq 1$ and obtain

$$
\begin{aligned}
\left|f_{n}(z)\right| & \leqq \int_{0}^{1}|1+(z-1) t|^{n}|d \chi(t)| \\
& =\int_{0}^{t_{1}}|1+(z-1) t|^{n}|d \chi(t)| \leqq \int_{0}^{t_{1}}|d \chi(t)| .
\end{aligned}
$$

To prove necessity, we simplify writing by setting $\zeta=z-1$, and assume that the sequence $f_{n}$ defined by

$$
f_{n}=\int_{0}^{1}(1+\zeta t)^{n} d \chi(t)
$$

(2) In case $H(\chi)$ is an Euler method $E_{r}$ for which $0<r<1, f_{n}(z)=[1+(z-1) r]^{n}$ and we see immediately that $f_{n}(z)$ is bounded if and only if $|1+(z-1) r| \leqq 1$ and hence if and only if $0<r \leqq t_{1}$ and therefore if and only if $\chi(t)=1$ over $t_{0}<t \leqq 1$. The case in which $H(\chi)$ is not an Euler method is not so simple. 
is bounded. Integrating by parts, setting

$$
u(t)=(1+\zeta t)^{n}, \quad v_{1}(t)=-\int_{t}^{1} d \chi(s)=\chi(t)-\chi(1)
$$

we obtain

$$
f_{n}=\int_{0}^{1} d \chi(t)-n \zeta \int_{0}^{1}(1+\zeta t)^{n-1} v_{1}(t) d t
$$

If we show that $v_{1}(t)=0$ over $t_{1}<t \leqq 1$, our result will follow. The function $v_{1}(t)$ has bounded variation over $0 \leqq t \leqq 1$ and has no removable discontinuities. If we set

$$
I_{n}^{(1)}=\int_{0}^{1}(1+\zeta t)^{n} v_{1}(t) d t
$$

then (3.11) and our hypotheses imply that $I_{n}^{(1)} \rightarrow 0$ as $n \rightarrow \infty$. Integrating by parts, setting

we obtain

$$
u=(1+\zeta t)^{n}, \quad v_{2}(t)=-\int_{t}^{1} v_{1}(u) d u
$$

$$
I_{n}^{(1)}=\int_{0}^{1} v_{1}(t) d t-n \zeta \int_{0}^{1}(1+\zeta t)^{n-1} v_{2}(t) d t
$$

If we show that $v_{2}(t)=0$ over $t_{1}<t \leqq 1$, our result will follow. The function $v_{2}(t)$ is continuous and if we set

$$
I_{n}^{(2)}=\int_{0}^{1}(1+\zeta t)^{n} v_{2}(t) d t
$$

then $I_{n}^{(2)} \rightarrow 0$ as $n \rightarrow \infty$. If we integrate by parts once more, setting

$$
g(t)=-\int_{t}^{1} v_{2}(u) d u
$$

we see that our result is a consequence of the following lemma.

LEMMA 3.2. If $g(t)$ has a continuous derivative, if $|1+\zeta|>1$, and if

$$
I_{n}=\int_{0}^{1}(1+\zeta t)^{n} g(t) d t, \quad n=0,1,2, \cdots,
$$

is bounded, then $g(t)=0$ over the interval $t_{1}<t \leqq 1$ of values of $t$ for which $|1+\zeta t|>1$.

Since $I_{n}$ is bounded, the series $\sum I_{n} w^{n}$ converges and defines a function 


$$
F_{1}(w)=\sum_{n=0}^{\infty} I_{n} w^{n}
$$

analytic over the open circular region $|w|<1$ of a complex $w$-plane. If $|w|<1 /|1+\zeta|$, then

$$
|w(1+\zeta t)| \leqq|w||1+\zeta|<1, \quad 0 \leqq t \leqq 1,
$$

so that

$$
\sum_{n=0}^{\infty}(1+\zeta t)^{n} w^{n} g(t)
$$

converges uniformly over $0 \leqq t \leqq 1$. Hence it follows from (3.21) that when $|w|<1 /|1+\zeta|$

$$
\sum_{n=0}^{\infty} I_{n} w^{n}=\int_{0}^{1} \frac{g(t)}{1-w-w \zeta t} d t .
$$

But the function $F_{2}(w)$ defined by

$$
F_{2}(w)=\int_{0}^{1} \frac{g(t)}{1-w-w \zeta t} d t
$$

is analytic at each point $w$ of the complex plane except possibly those for which the equation

$$
w=\frac{1}{1+\zeta t}
$$

holds for some $t$ in the interval $0 \leqq t \leqq 1$.

Let $t_{0}$ be fixed such that $0<t_{0} \leqq 1$ and $\left|1+\zeta t_{0}\right|>1$. If we set, for $y$ real,

then

$$
w_{y}=\frac{1}{1+\zeta\left(t_{0}+i y\right)}
$$

$$
1-w_{y}(1+\zeta t)=1-\frac{1+\zeta t}{1+\zeta\left(t_{0}+i y\right)}=\frac{\zeta\left(t_{0}-t+i y\right)}{1+\zeta\left(t_{0}+i y\right)}
$$

and hence when $y \neq 0$

$$
F_{2}\left(w_{y}\right)=-\frac{1+\zeta\left(t_{0}+i y\right)}{\zeta} \int_{0}^{1} \frac{g(t)}{t-t_{0}-i y} d t .
$$

But $F_{1}(w)$ and $F_{2}(w)$ are functions which are analytic and equal at points $w=w_{y}$ for which $y$ is real, not 0 , and $|y|$ is sufficiently small. Moreover $F_{1}(w)$ is analytic at the point $w=w_{0} \equiv 1 /\left(1+\zeta t_{0}\right)$. Therefore $\lim _{y \rightarrow 0} F_{2}\left(w_{y}\right)$ must exist. Hence if we set 


$$
G(y)=\int_{0}^{1} \frac{g(t)}{t-t_{0}-i y} d t, \quad y \neq 0,
$$

then $\lim _{y \rightarrow 0} G(y)$ must exist. We are now in a position to complete the proof by showing that $g\left(t_{0}\right)$ must be 0 .

Since $g(t)$ has a continuous derivative, there is a continuous function $B(t)$ such that

$$
g(t)=B(t)\left(t-t_{0}\right)+g\left(t_{0}\right) .
$$

Hence $G(y)=G_{1}(y)+G_{2}(y)$ where

$$
G_{1}(y)=\int_{0}^{1} \frac{B(t)\left(t-t_{0}\right)}{t-t_{0}-i y} d t, \quad G_{2}(y)=\int_{0}^{1} \frac{g\left(t_{0}\right)}{t-t_{0}-i y} d t .
$$

Since the first integrand is dominated by $|B(t)|$ and converges to $B(t)$ as $y \rightarrow 0, \lim _{y \rightarrow 0} G_{1}(y)$ exists; hence $\lim _{y \rightarrow 0} G_{2}(y)$ must exist. Upon evaluating the integral for $G_{2}(y)$, we see that existence of $\lim _{y \rightarrow 0} G_{2}(y)$ implies that $g\left(t_{0}\right)=0$. This completes the proof of Lemma 3.2 and hence also the proofs of Theorems 3.1 and 1.1 .

4. Uniform summability of power series inside Euler polygons $B(r)$. Let $\sum c_{n} z^{n}$ be a power series with a finite positive radius of convergence $R$, and let $f(z)$ be the function generated by analytic extension along radial lines from the origin. The open set in which $f(z)$ is thus defined is the Mittag Leffler star $S$. If a half-line $l_{\phi}$ of points $z$, such that $z=\rho e^{i \phi}$ where $\rho \geqq 0$, contains no singular point of $f(z)$, then $l_{\phi}$ is in $S$. If $l_{\phi}$ contains a singular point, and $\rho_{0}$ is the least value of $\rho$ for which $\rho e^{i \phi}$ is a singular point, then the point $\zeta=\rho_{0} e^{i \phi}$ is a vertex of the star; the points of $l_{\phi}$ for which $0 \leqq \rho<\rho_{0}$ lie in $S$ and the points for which $\rho \geqq \rho_{0}$ are exterior to $S$.

Let $r$ be fixed such that $0<r \leqq 1$. Corresponding to each vertex $\zeta$ of the star, let $B(r, \zeta)$ denote the set of points $z$ for which

$$
\left|z-\left(1-\frac{1}{r}\right) \zeta\right|<\frac{|\zeta|}{r}
$$

This set $B(r, \zeta)$ is the interior of the circle, with center at $\left(1-r^{-1}\right) \zeta$, which passes through the point $\zeta$. The set $B(r, \zeta)$ contains the interior of the circle of convergence. Let $B(r)$ denote the set of inner points of the intersection of the sets $B(r, \zeta)$ determined by the set of vertices $\zeta$ of $S$. This set $B(r)$, which is not a polygon in the ordinary sense, was called a curvilinear polygon by Knopp [14]; we shall call it the Euler polygon of order $r$. For each $r, B(r)$ is a bounded convex open set containing the inner points of the circle of convergence. If $r_{1}<r_{2}$, then $B\left(r_{2}, \zeta\right) \subset B\left(r_{1}, \zeta\right)$ for each $\zeta$ and accordingly $B\left(r_{2}\right) \subset B\left(r_{1}\right)$. The union, for $0<r \leqq 1$ of the sets $B(r)$ is (Knopp [14] and Rademacher [18]) the Borel polygon $B$. It was shown by Knopp and Rademacher, for the case 
in which $r=2^{-p}$, that $\sum c_{n} z^{n}$ is summable $E_{r}$ or non-summable $E_{r}$ according as $z$ lies inside or outside the Euler polygon $B_{r}$ of order $r$. The following theorem presents a fact, involving summability by regular Hausdorff methods, which is the analogue of the fundamental fact that a power series having a finite radius of convergence converges uniformly over each closed set $F$ inside the circle of convergence.

THEOREM 4.1. If $H(\chi)$ is a regular Hausdorf transformation of order $r$ and $F$ is a closed subset of the Euler polygon $B(r)$ of order $r$ of a power series $\sum c_{n} z^{n}$ having a finite positive radius of convergence, then $\sum c_{n} z^{n}$ is summable $H(\chi)$ uniformly over $F$ to the function $f(z)$ obtained by analytic extension of $\sum c_{n} z^{n}$ along radial lines from the origin.

Let $z_{1} \in B(r)$.Then, when $\zeta$ is a vertex of the star $S,\left|z_{1}-\left(1-r^{-1}\right) \zeta\right|<r^{-1} \zeta$. Let $\zeta^{\prime}$ be a point not in $S$. Then a vertex $\zeta$ of $S$ and a number $\rho \geqq 1$ exist such that $\zeta^{\prime}=\rho \zeta$. The circular set of points $z$ for which $\left|z-\left(1-r^{-1}\right) \zeta^{\prime}\right|<r^{-1}\left|\zeta^{\prime}\right|$ contains the circular set of points $z$ for which $\left|z-\left(1-r^{-1}\right) \zeta\right|<r^{-1}|\zeta|$ and hence contains $z_{1}$. This shows that if $z_{1} \in B(r)$ and $\zeta^{\prime}$ is not in the star, then $\left|z_{1}-\left(1-r^{-1}\right) \zeta^{\prime}\right|<r^{-1}\left|\zeta^{\prime}\right|$. It follows that if $z_{1} \in B(r)$, then the set of all poin ts $u$ for which

$$
\left|z_{1}-\left(1-r^{-1}\right) u\right| \geqq r^{-1}|u|
$$

must lie in the star. The set of points $u$ for which (4.11) holds is the set for which

$$
\left|u-\frac{1-r}{2-r} z_{1}\right| \leqq \frac{\left|z_{1}\right|}{2-r} .
$$

Therefore, if $z_{1} \in B(r)$, the circular set of points $u$ satisfying (4.11) and (4.12) must lie in the star $S$. This circular set contains the origin in its interior, and the point $z_{1}$ lies on the boundary.

Let $\rho(\phi)$ be the continuous positive function such that the point $w=\rho(\phi) e^{i \phi}$ traverses the boundary of $B(r)$ as $\phi$ increases from 0 to $2 \pi$. Corresponding to each multiplier $h$ for which $0<h<1$, let $\beta(r, h)$ be the open set of points inside the curve $\omega=h \rho(\phi) e^{i \phi}$. The sets $\beta(r, h)$ are nested subsets of $B(r)$ in the sense that if $0<h_{1}<h_{2}<1$, then $\bar{\beta}\left(r, h_{1}\right) \subset \beta\left(r, h_{2}\right) \subset B(r)$. Since $F$ is a closed subset of the open set $B(r)$, there is a multiplier $h_{0}$ such that $0<h_{0}<1$ and $F \subset \beta\left(r, h_{0}\right)$. Let $h_{1}$ be fixed such that $h_{0}<h_{1}<1$. Since $H(\chi)$ is regular, the $H(\chi)$ transform of $\sum c_{n} z^{n}$ converges to $f(0)$ when $z=0$. Accordingly, it is suffcient to prove that the $H(\chi)$ transform of $\sum c_{n} z^{n}$ converges to $f(z)$ uniformly over the set $\beta_{0}$ obtained by deleting the point $z=0$ from the set $\beta\left(r, h_{0}\right)$.

Let $z \in \beta_{0}$. Then there is a number $\lambda \equiv \lambda(z)>1$ such that the point $\lambda z$ lies on the boundary of the set $\beta\left(r, h_{1}\right)$; we shall want to use the fact that $\lambda>\lambda_{0}$ where $\lambda_{0}=h_{1} / h_{0}$. Let $C(z)$ denote the circle composed of the points $u$ for which 


$$
\left|\lambda z-\left(1-r^{-1}\right) u\right|=r^{-1}|u| \text {. }
$$

Since $\lambda z \in B(r)$, the circle $C(z)$ lies in the star. The equation of $C(z)$ can be written in the form

$$
\left|u-\frac{1-r}{2-r} \lambda z\right|=\frac{|\lambda z|}{2-r} .
$$

From this equation, it is apparent that the origin and the point $z$ lie inside the circle $C(z)$; in fact 0 and $z$ are points of the diameter having its ends at the points $-[r /(2-r)] \lambda z$ and $\lambda z$. Since $0<r /(2-r)<1<\lambda$, it is apparent that if $u \in C(z)$, then

$$
|u| \geqq \frac{r}{2-r}|\lambda z| \geqq \frac{r c_{1}}{2-r}=c_{2}>0
$$

where $c_{1}$ is the minimum value of $|\lambda z|$ when $\lambda z$ lies on the boundary of the set $\beta\left(r, h_{1}\right)$ and accordingly $c_{1}=h_{1} R$ where $R$ is the radius of convergence of $\sum c_{n} z^{n}$; and where $c_{2}$ is defined by the last equality. Since on the one hand $|\lambda z-z| \geqq c_{3}>0$ where $c_{3}$ is the minimum value of $\left|z_{1}-z_{2}\right|$ when $z_{1}$ lies on the boundary of the set $\beta\left(r, h_{1}\right)$ and $z_{2}$ lies in the closure of the set $\beta\left(r, h_{0}\right)$, and on the other hand

$$
|z-[-r /(2-r)] \lambda z| \geqq|[r /(2-r)] \lambda z| \geqq c_{2},
$$

it follows that when $u \in C(z)$

$$
|u-z| \geqq c_{4}>0
$$

the constant $c_{4}$ being the minimum value of $c_{3}$ and $c_{2}$.

When $z \in \beta_{0}$ and $u \in C(z)$, the equality (4.21) gives

$$
\left|\frac{z}{u}-\frac{1}{\lambda}\left(1-\frac{1}{r}\right)\right|=\frac{1}{\lambda} \cdot \frac{1}{r} .
$$

If $\mu_{1} \leqq \mu_{0}$, then the set of points $v$ of the complex plane for which the inequality

$$
\left|v-\mu\left(1-r^{-1}\right)\right| \leqq \mu r^{-1}
$$

holds when $\mu=\mu_{1}$ is a subset of the set of points $v$ for which the inequality holds when $\mu=\mu_{0}$. Hence we can use (4.31) and the fact that $\lambda \equiv \lambda(z) \geqq \lambda_{0}$ to obtain

$$
\left|\frac{z}{u}-\frac{1}{\lambda_{0}}\left(1-\frac{1}{r}\right)\right| \leqq \frac{1}{\lambda_{0}} \cdot \frac{1}{r} .
$$

When $\mu$ has the fixed value $\mu=\lambda_{0}^{-1}<1$, the set of points $v$ for which (4.32) holds is a closed subset of the set of points $v$ for which

$$
\left|v-\left(1-r^{-1}\right)\right|<r^{-1} \text {. }
$$


Hence there is a constant $\theta$, depending only on $r$ and $\lambda_{0}$ and independent of $z$ and $u$, such that $0<\theta<1$ and

$$
\left|\frac{z}{u}-\left(1-\frac{1}{r}\right)\right| \leqq \frac{\theta}{r} .
$$

If $\delta$ is a fixed number for which $0<\delta<r$, then (4.33) implies existence of $\alpha$ and $\phi$ such that $0<\alpha \leqq 1,0<\phi \leqq 2 \pi$ and

$$
\frac{z}{u}=\left(1-\frac{1}{r}\right)+\frac{\alpha \theta}{r} e^{i \phi}
$$

and it follows easily that, when $\delta \leqq t \leqq r$,

$$
\begin{aligned}
\left|1+t\left(\frac{z}{u}-1\right)\right| & =\left|1-\frac{t}{r}+\frac{\alpha t \theta}{r} e^{i \phi}\right| \leqq 1-t\left(\frac{1-\theta}{r}\right) \\
& \leqq 1-\delta\left(\frac{1-\theta}{r}\right)=\theta_{1}<1
\end{aligned}
$$

where $\theta_{1}$ is a constant, defined by the equality, depending on $\delta, \theta$, and $r$ but independent of $z$ and $u$. These considerations show also that

$$
\left|1+t\left(\frac{z}{u}-1\right)\right| \leqq 1, \quad 0 \leqq t \leqq r .
$$

For each $h$ in the interval $0<h<1$, let $W(h)$ denote the set which contains those and only those points $w$ for which the inequality

$$
\left|z^{\prime}-\left(1-r^{-1}\right) w\right| \geqq r^{-1}|w|
$$

holds for at least one point $z^{\prime}$ in the closure of the set $\beta(r, h)$ defined above. For each $h, W(h)$ is [see (4.11)] the union of sets in the star and accordingly $W(h)$ is in the star. It is probably true that $W(h)$ is closed, but we do not need the result. For each $h$, the set $W(h)$ is bounded. If $0<h_{1}<h_{2}<1$, then the points of the closure of $W\left(h_{1}\right)$ are inner points of $W\left(h_{2}\right)$ and it follows that the closure $\bar{W}\left(h_{1}\right)$ of $W\left(h_{1}\right)$ lies in the star. Since $\bar{W}\left(h_{1}\right)$ is a bounded closed subset of the star, $f(z)$ must be bounded over $\bar{W}\left(h_{1}\right)$. Choose a constant $c_{5}$ such that

$$
|f(z)| \leqq c_{5}, \quad z \in \bar{W}\left(h_{1}\right) .
$$

From the definitions of $C(z)$ and $W\left(h_{1}\right)$, it follows that the points on the curve $C(z)$ lie in the set $W\left(h_{1}\right)$ for each $z \in \beta_{0}$. Hence, when $z \in \beta_{0}$,

$$
|f(u)| \leqq c_{5}
$$$$
u \in C(z)
$$

the constant $c_{5}$ being independent of $z$ and $u$. 
For each $z \in \beta_{0}$, let $C(z)$ be the circle defined above. The coefficients $c_{j}$ are related to $f(z)$ by the familiar formula

$$
c_{j}=\frac{1}{2 \pi i} \int_{C(z)} \frac{f(u)}{u^{j+1}} d u, \quad j=0,1,2, \cdots
$$

and the fact that $C(z)$ surrounds both the origin and the point $z$ implies that the partial sums of $\sum c_{j} z^{j}$ are given by

$$
\begin{aligned}
S_{k}(z) & =\frac{1}{2 \pi i} \int_{C(z)} \frac{f(u)}{u-z}\left[1-\left(\frac{z}{u}\right)^{k+1}\right] d u \\
& =f(z)-\frac{1}{2 \pi i} \int_{C(z)} \frac{z f(u)}{u(u-z)}\left(\frac{z}{u}\right)^{k} d u .
\end{aligned}
$$

The $H(\chi)$ transform of $\sum c_{j} z^{j}$ is accordingly given by

$$
\sigma_{n}(z)=f(z)-(2 \pi i)^{-1} R_{n}(z)
$$

where

$$
\begin{aligned}
R_{n}(z) & =\int_{0}^{1} d \chi(t) \int_{C(z)} \frac{z f(u)}{u(u-z)} \sum_{k=0}^{n} C_{n, k}\left(\frac{t z}{u}\right)^{k}(1-t)^{n-k} d u \\
& =\int_{0}^{1} d \chi(t) \int_{C(z)} \frac{z f(u)}{u(u-z)}\left[1+t\left(\frac{z}{u}-1\right)\right]^{n} d u
\end{aligned}
$$

Hence we may complete the proof of Theorem 4.1 by showing that $R_{n}(z)$ converges to 0 uniformly over $\beta_{0}$. Since $\chi(t)$ is constant over $r<t \leqq 1$, we may assume that $\chi(t)=1$ over $r \leqq t \leqq 1$ and replace the upper limit of integration by $r$. We then obtain

$$
\left|R_{n}(z)\right| \leqq \int_{0}^{r}|d \chi(t)| \int_{C(z)} \frac{|z||f(u)|}{|u||u-z|}\left|1+t\left(\frac{z}{u}-1\right)\right|^{n}|d u| .
$$

If $c_{6}$ denotes the least upper bound of $|z|$ for $z \in \beta_{0}$, then

$$
\frac{|z||f(u)|}{|u||u-z|} \leqq \frac{c_{6} c_{5}}{c_{2} c_{4}}=c_{7}
$$

where $c_{7}$ is the constant, independent of $z$ and $u$, defined by the last equality. Let $c_{8}$ be the least upper bound of the circumferences of the circles $C(z)$; it is obviously finite since the circles lie in the bounded set $W\left(h_{1}\right)$. Let $c_{9}=c_{7} c_{8}$. With the aid of (4.34) and (4.35) we obtain, when $0<\delta<r$

$$
\left|R_{n}(z)\right| \leqq c_{9} \int_{0}^{\delta}|d \chi(t)|+c_{9} \int_{\delta}^{r} \theta_{1}^{n}|d \chi(t)| .
$$

Let $\epsilon>0$, and fix $\delta$ such that $0<\delta<r$ and the first term on the right is less than $\epsilon / 2$. Then choose $N$ such that the last term is less than $\epsilon / 2$ when $n \geqq N$. 
We then have $\left|R_{n}(z)\right|<\epsilon$ when $z \in \beta_{0}$ and $n \geqq N$. This completes the proof of Theorem 4.1 .

Throughout this section, we have considered power series $\sum c_{n} z^{n}$ for which the radius of convergence $R$ is finite. In case $R=\infty$ the series is uniformly summable, to the entire function $f(z)$ determined by the series, over each bounded set $E$ by each regular transformation of the form

$$
\sigma(t)=\sum_{k=0}^{\infty} a_{k}(t) s_{k}
$$

and hence in particular by each regular transformation of the form $H(\chi)$. To prove this, we note first that if $s_{n}(z), n=0,1,2, \cdots$, is the sequence of partial sums of $\sum c_{n} z^{n}$, then the sequence converges uniformly over $E$ to $f(z)$ and the sequence is uniformly bounded over $E$. Hence (see Agnew [1, Theorem 7.21], and Agnew [2]) the sequence $s_{n}(z)$ and the series $\sum c_{n} z^{n}$ must be uniformly summable to $f(z)$ over $E$.

5. Other methods of summability. Let $\sum c_{n} z^{n}$ be a power series having a finite positive radius of convergence, and let $F$ be a closed set interior to the Borel polygon $B$. Then $r$ exists such that $0<r \leqq 1$ and $F \subset B(r)$, and, by Theorem $4.1, \sum c_{n} z^{n}$ is uniformly summable $E_{r}$ over $F$ to $f(z)$. Let $\sigma_{n}^{(r)}(z)$, $n=0,1, \cdots$, denote the $E_{r}$ transform of $\sum c_{n} z^{n}$. Since $f(z)$ and the functions $\sigma_{1}^{(r)}(z), \sigma_{2}^{(r)}(z), \cdots$ are each bounded over $F$, it follows that the sequence $\sigma_{n}^{(r)}(z)$ is uniformly bounded over $F$.

Let $G$ be a method of summability of the form (4.7) which includes $E_{r}$. For example, $G$ may be the Borel exponential method (Hurwitz [12, p. 27]) or the LeRoy method (Morse [15, p. 281]). Let $G$ be such that, for each $t$,

$$
\sum_{k=0}^{\infty} a_{k}(t) z^{k}
$$

converges for all complex values of $z$. This requirement, which is automatically satisfied when $G$ is of finite reference, is also satisfied when $G$ is either the Borel exponential method or the LeRoy method. Since the inverse of $E_{r}$ is $E_{1 / r}$ (Hurwitz [12]), the $G$ transform of $s_{n}(z)$ may be written

$$
\sigma(t, z)=\sum_{k=0}^{\infty} \sum_{p=0}^{k} a_{k}(t) C_{k, p} \frac{1}{r^{p}}\left(1-\frac{1}{r}\right)^{k-p} \sigma_{p}^{(r)}(z) .
$$

Our hypotheses imply that, for each $t$, the series in the right member of (5.1) is absolutely convergent. Hence (5.1) can be written in the form

$$
\tilde{\sigma}(t)=\sum_{p=0}^{\infty}\left[\sum_{k=p}^{\infty} a_{k}(t) C_{k, p} \frac{1}{r^{p}}\left(1-\frac{1}{r}\right)^{k-p}\right] \tilde{s}_{p}
$$

where $\tilde{\sigma}(t)=\sigma(t, z)$ and $\tilde{s}_{p}=\sigma_{p}^{(r)}(z)$. The hypothesis that $G$ includes $E_{r}$ im- 
plies that (5.2) is a regular transformation of the form (4.7). Hence it follows, as at the end of the last section, that $\sigma(t, z)$ must converge to $f(z)$ uniformly over $F$, that is, $\sum c_{n} z^{n}$ is summable $G$ to $f(z)$ uniformly over $F$. In particular, a power series $\sum c_{n} z^{n}$ is summable by the Borel exponential method uniformly over each bounded closed set inside the Borel polygon. This particular result may be known, but the author is unable to give a reference. Borel [4] and Phragmén [16] have shown that $\sum c_{n} z^{n}$ is summable, by the exponential method, at each point inside the Borel polygon and non-summable at each point outside the polygon; and Doetsch [5] has discussed summability on the boundary of the polygon.

6. Collective Hausdorff summability $\mathfrak{H}$. It was shown by Hurwitz and Silverman [13] that a transformation of the form

$$
\sigma_{n}=\sum_{k=0}^{\infty} a_{n k} s_{k}
$$

commutes with the $C_{1}$ transformation

$$
\sigma_{n}=\frac{1}{n+1} \sum_{k=0}^{n+1} s_{k}
$$

if and only if it has the form

$$
\sigma_{n}=\sum_{k=0}^{n} C_{n, k}\left\{\sum_{j=k}^{n}(-1)^{j-k} C_{m}-k, j-k \lambda_{j}\right\} s_{k}
$$

where $\lambda_{0}, \lambda_{1}, \cdots$ is a sequence of complex constants. It was shown by Hausdorff [9] that such a transformation is regular if and only if the sequence $\lambda_{0}, \lambda_{1}, \cdots$ is the moment sequence

$$
\lambda_{n}=\int_{0}^{1} t^{n} d \chi(t)
$$

of a function $\chi(t)$, having bounded variation over $0 \leqq t \leqq 1$, such that $\chi(0+)=\chi(0)=0$ and $\chi(1)=1$. When (6.14) holds, the transformation (6.13) takes the form $H(\chi)$. As was shown both by Hurwitz and Silverman and by Hausdorff, these regular transformations commute with each other as well as with $C_{1}$ and hence constitute a system of consistent methods of summability. By this we mean that if a series or sequence is summable by two different regular methods of the form $H(\chi)$, then the two values assigned must be equal.

This circumstance makes possible the following definition of a method $\mathfrak{F}$ of summability which makes use of the collection of regular methods $H(\chi)$ and which may be called the collective Hausdorff method. Let a series $\sum u_{n}$ be called summable $\mathfrak{F}$ to the value $\sigma$ if it is summable to the value $\sigma$ by at least one regular method $H(\chi)$. 
The method $\mathcal{H C}$ is obviously regular. It is also linear; by this we mean that if two series $\sum u_{n}$ and $\sum v_{n}$ with partial sums $s_{n}$ and $t_{n}$ are summable $\mathfrak{F}$ to $U$ and $V$, respectively, and if $\alpha$ and $\beta$ are constants, then $\sum\left(\alpha u_{n}+\beta v_{n}\right)$ is summable $\mathfrak{H C}$ to $\alpha U+\beta V$. This follows from Hurwitz and Silverman [13, Theorem 3].

It is clear from Theorem 1.1 that the method $\mathfrak{F}$ is stronger than any one method of the form $H(\chi)$; if $H(\chi)$ is regular, then $\sum z^{n}$ is summable $H(\chi)$ only inside and perhaps at some of the points on the boundary of the appropriate circle of summability, but $\sum z^{n}$ is summable $\mathfrak{H}$ for each $z$ in the halfplane $R z<1$. It would be interesting to know whether there is a regular method of summability, based on a single sequence-to-sequence or sequenceto-function transformation of the familiar type, which includes $\mathfrak{H}$; perhaps there is one which is equivalent to $\mathcal{F}$. The Borel exponential method $B_{1}$ evaluates $\sum z^{n}$ when $R(z)<1$; but $B_{1}$ does not include the method $C_{1}$ determined by $\chi(t)=t$ and hence $B_{1}$ does not include $\mathcal{F}$. The LeRoy method $L$ includes the regular Euler methods $E_{r}$ (Morse [15]) and the regular Cesàro methods $C_{r}$ (Garabedian [6]); but, if one may judge from the difficulty in showing that $L$ includes $C_{r}$, it must be difficult to determine the extent to which $L$ includes Hausdorff methods. That $L$ does not include $\mathfrak{F C}$ is a corollary of Theorem 8.4.

It follows from Theorem 1 that the series $\sum z^{n}$ is non-summable $\mathcal{H}$ for each $z$ for which $R z>1$. This result creates a strong presumption that $\mathcal{F C}$ is ineffective outside the Borel polygon of a power series $\sum a_{n} z^{n}$. If a point $z_{0}$ lies outside the Borel polygon of a series $\sum a_{n} z^{n}$, then $z_{0}$ lies outside the circle of convergence and accordingly the series $\sum a_{n} z_{0}^{n}$ has unbounded partial sums.

We now show existence of series with bounded partial sums $s_{n}$ which are not summable $\mathfrak{H}$. The series are gap series with large gaps. Let $n_{0}, n_{1}, n_{2}, \cdots$ be a sequence of integers for which $0=n_{0}<n_{1}<n_{2}<\cdots$ and

$$
n_{p+1} / n_{p} \rightarrow \infty
$$

as $p \rightarrow \infty$. Let $b_{1}, b_{2}, \cdots$ be a bounded divergent sequence of complex numbers, and let $\sum u_{n}$ be the series whose partial sums $s_{0}, s_{1}, \cdots \cdot$ are defined by the formulas

$$
s_{k}=b_{p}, \quad n_{p-1} \leqq k \leqq n_{p} ; p=1,2, \cdots .
$$

Let $H(\chi)$ be regular; we show that $\sum u_{n}$ is non-summable $\mathfrak{F}$ by showing that it is non-summable $H(\chi)$. Letting $\sigma_{n}$ denote the $H(\chi)$ transform of $\sum u_{n}$, and setting $m_{p}=n_{p}-1$, we find that when $p>1$

and

$$
\sigma\left(m_{p}\right)=\int_{0}^{1} \sum_{k=0}^{m_{p}} C_{m_{p}, k} t^{k}(1-t)^{m_{p}-k_{s}} s_{k} \chi(t)
$$




$$
\begin{aligned}
\sigma\left(m_{p}\right)-s\left(m_{p}\right) & =\int_{0}^{1} \sum_{k=0}^{m_{p}} C_{m_{p}, k} t^{k}(1-t)^{m_{p}-k}\left[s_{k}-s\left(m_{p}\right)\right] d \chi(t) \\
& =\int_{0}^{1} \sum_{k=0}^{m_{\eta-1}} C_{m_{p}, k} t^{k}(1-t)^{m_{p}-k}\left[s_{k}-s\left(m_{p}\right)\right] d \chi(t)
\end{aligned}
$$

so that, where $M$ is a constant for which $\left|b_{k}\right| \leqq M$ for each $k=0,1,2, \cdots$,

$$
\left|\sigma\left(m_{p}\right)-s\left(m_{p}\right)\right| \leqq 2 M \int_{0}^{1} \sum_{k=0}^{m_{n-1}} C_{m_{p}, k} t^{k}(1-t)^{m_{p}-k}|d \chi(t)| .
$$

Let $\epsilon>0$. Choose $\delta>0$ such that

$$
2 M \int_{0}^{\delta}|d \chi(t)|<\epsilon / 2 .
$$

Let $0<\theta<\delta$ and choose an index $P$ such that $m_{p-1}<\theta m_{p}$ when $p \geqq P$. Then when $p \geqq P$

$$
\left|\sigma\left(m_{p}\right)-s\left(m_{p}\right)\right|<\epsilon / 2+2 M \int_{\delta}^{1} \sum_{k<\theta m_{p}} C_{m_{p}, k} t^{k}(1-t)^{m_{p}-k}|d \chi(t)| .
$$

If $L_{1}, L_{2}, \cdots$ is a sequence of positive constants for which $L_{n} \rightarrow \infty$ and and $L_{n} / n \rightarrow 0$, say $L_{n}=n^{1 / 2}$, we can use the elementary inequality (see, for example, Hausdorff $[9$, p. 104])

$$
\sum_{k<k(n, t)} C_{n, k} t^{k}(1-t)^{n-k} \leqq \frac{1}{4 L_{n}}
$$

where $k(n, t)=n\left\{t-\left(L_{n} / n\right)^{1 / 2}\right\}$, to obtain, when $p$ is sufficiently great,

$$
\left|\sigma\left(m_{p}\right)-s\left(m_{p}\right)\right|<\frac{\epsilon}{2}+\frac{2 M}{4 L\left(m_{p}\right)} \int_{\delta}^{1}|d \chi(t)| .
$$

Since the last term is less than $\epsilon / 2$ when $p$ is sufficiently great, and since $s\left(m_{p}\right)=b_{p}$, this implies that

$$
\lim _{p \rightarrow \infty}\left|\sigma\left(m_{p}\right)-b_{p}\right|=0 .
$$

Thus divergence of the sequence $b_{n}$ implies that of the sequence $\sigma_{n}$ and accordingly $\sum u_{n}$ is non-summable $H(\chi)$.

7. A Tauberian theorem for Hausdorff methods. It is easy to amplify the work of the preceding paragraph to establish the following Tauberian gap theorem in which there is no order condition placed upon the non-vanishing terms or partial sums of the series.

THEOREM 7.1. If $n_{0}, n_{1}, n_{2}, \cdots$ is a sequence of integers for which $0=n_{0}<n_{1}<n_{2}<\cdots$ and 


$$
n_{p+1} / n_{p} \rightarrow \infty
$$

as $p \rightarrow \infty$, if $\sum u_{n}$ is a series for which

$$
u_{n}=0 \text {, }
$$

$n \neq n_{0}, n_{1}, n_{2}, \cdots$,

and if $\sum u_{n}$ is summable $\mathfrak{H}$, then $\sum u_{n}$ is convergent $\left({ }^{3}\right)$.

The hypothesis implies existence of a sequence $b_{1}, b_{2}, \cdots$ such that the partial sums $s_{0}, s_{1}, \cdots$ of $\sum u_{n}$ satisfy (6.21). Let $H(\chi)$ be a regular Hausdorff method by which $\sum u_{n}$ is summable. In case the sequence $b_{n}$ is bounded, we can proceed exactly as above to obtain (6.4). Convergence of $\sigma_{n}$ then implies that of $b_{n}$ and hence that of $\sum u_{n}$. It remains for us to show that the sequence $b_{k}$ must be bounded. For each $p=1,2, \cdots$ let $M_{p}$ denote the maximum value of $\left|s_{k}\right|$ when $0 \leqq k \leqq m_{p}$ where, as above, $m_{p}=n_{p}-1$. Then we can obtain (6.3) and conclude that

$$
\left|\sigma\left(m_{p}\right)-s\left(m_{p}\right)\right| \leqq 2 M_{p} \int_{0}^{1} \sum_{k=0}^{m_{p}-1} C_{m_{p}, k} t^{k}(1-t)^{m_{p}-k}|d \chi(t)| .
$$

Choose $\delta>0$ such that

$$
\int_{0}^{\delta}|d \chi(t)|<1 / 5
$$

and then choose an index $P$ such that

$$
\int_{\delta}^{1} \sum_{k=0}^{m_{p-1}} C_{m_{p}, k} t^{k}(1-t)^{m_{p}-k}|d \chi(t)|<1 / 5, \quad p \geqq P .
$$

Then

$$
\left|\sigma\left(m_{p}\right)-s\left(m_{p}\right)\right| \leqq(4 / 5) M_{p}, \quad p \geqq P .
$$

If the sequence $b_{k}$ is unbounded, then $M_{p} \rightarrow \infty$ as $p \rightarrow \infty$ and there is an infinite set of indices $p \geqq P$ for which $\left|s\left(m_{p}\right)\right|=M_{p}$. For such values of $p$, $\left|\sigma\left(m_{p}\right)\right| \geqq M_{p} / 5$. This is inconsistent with the hypothesis that $\sum u_{n}$ is summable $H(\chi)$; hence the sequence $b_{k}$ must be bounded and Theorem 7.1 is proved.

8. Criteria involving zeros of moment functions. Let $H(\chi)$ be regular, and let

$$
\mu(z)=\int_{0}^{1} t^{z} d \chi(t), \quad z \geqq 0,
$$

be the moment function determined by $\chi(t)$. The function $\mu(z)$ is continuous in the closed half-plane $R z \geqq 0$ and is analytic in the open half-plane $R z>0$.

(3) For a Tauberian theorem involving a subclass of the regular Hausdorff methods, see Pitt [17, pp. 280-284] and Agnew [3]. 
Several investigations have shown that the zeros of $\mu(z)$ play a fundamental role in the theory of transformations of the form $H(\chi)$. This applies, in particular, to the relation between $H(\chi)$ and generalized Abel summability $A^{*}$ used by Silverman and Tamarkin [20]. Let a series $\sum u_{n}$ with partial sums $s_{n}$ be called summable $A^{*}$ to $\sigma^{*}$ if the series

$$
\sum_{k=0}^{\infty}(1-z) z^{k} s_{k}
$$

has.a positive radius of convergence and defines, by analytic extension along radial lines from the origin, a function $\sigma^{*}(z)$ such that $\sigma^{*}(z)$ exists when $0<z<1$ and $\sigma^{*}(z) \rightarrow \sigma^{*}$ as $z \rightarrow 1$ over the set $0<z<1$. It was shown by Silverman and Tamarkin [20] that if $\mu(z)$ has a zero with real part positive, then $A^{*}$ does not include $H(\chi)$; and that if $\mu(z)$ has a zero with real part 0 and a certain supplementary condition on $\chi(t)$ is satisfied, then again $A^{*}$ does not include $H(\chi)$. In this section we obtain some results involving zeros of $\mu(z)$ on the critical line $R z=0$; in particular we remove the supplementary condition of Silverman and Tamarkin by proving the following theorem.

THEOREM 8.1. If $\chi(t)$ is regular and if $\mu(z)$ has a zero $q$ for which $R q=0$, then $A^{*}$ does not include $H(\chi)$.

Our results are obtained from consideration of the sequence

$$
s_{k}^{(q)}=k^{q}=e^{q \log k}
$$

in which $q$ is a complex number with $R q \geqq 0$ and $q \neq 0$. Let $H(\chi)$ be regular, and let $\sigma_{n}^{(q)}$ denote the $H(\chi)$ transform of the sequence $s_{n}^{(\ell)}$. Then

$$
\sigma_{n}^{(q)} / n^{q}=\int_{0}^{1} f_{n}(t) d \chi(t)
$$

where

$$
f_{n}(t)=\sum_{k=0}^{n} C_{n, t} t^{k}(1-t)^{n-k} f(k / n)
$$

and

$$
f(t)=t^{q}
$$

The function $f(t)$ is bounded, is continuous over $\delta \leqq t \leqq 1$ for each $\delta>0$, and is continuous at $t=0$ if and only if $R q>0$. For each $n$, the function $f_{n}(t)$ is (see Hausdorff, [9, p. 104]) the $n$th Bernstein polynomial determined by $f(t)$, and $f_{n}(t)$ converges uniformly to $f(t)$ over each interval $0<\delta \leqq t \leqq 1$. Let $\epsilon>0$. Choose $\delta>0$ such that

$$
\int_{0}^{\delta}|d \chi(t)|<\epsilon / 2
$$


Then, since $\left|f_{n}(t)\right| \leqq 1$ and $|f(t)| \leqq 1$ when $0 \leqq t \leqq 1$ and $n=0,1,2, \cdots$,

$$
\begin{aligned}
\mid \int_{0}^{1} f_{n}(t) d \chi(t) & -\int_{0}^{1} f(t) d \chi(t) \mid \\
& \leqq \int_{0}^{\delta}\left|f_{n}(t)-f(t)\right||d \chi(t)|+\int_{\delta}^{1}\left|f_{n}(t)-f(t)\right||d \chi(t)| \\
& \leqq \epsilon+\left[\max _{\delta \leqq t \leqq 1}\left|f_{n}(t)-f(t)\right|\right] \int_{\delta}^{1}|d \chi(t)| .
\end{aligned}
$$

This implies that, as $n \rightarrow \infty$, the superior limit of the first member of (8.15) is less than or equal to $\epsilon$ and hence 0 . Hence we can let $n \rightarrow \infty$ in (8.11) to obtain

$$
\lim _{n \rightarrow \infty} \frac{\sigma_{n}^{(q)}}{n^{q}}=\int_{0}^{1} t^{q} d \chi(t)=\mu(q)
$$

Suppose now that $R q=0$ but $q \neq 0$, say $q=i y$ where $y$ is real and $y \neq 0$. Then the sequence

$$
s_{k}^{(q)}=k^{q}=e^{i v \log k}
$$

is a sequence of points, on the unit circle, having the unit circle for its set of limit points. If $\mu(q)=0$, then (8.16) implies that $\sigma_{n}^{(q)} \rightarrow 0$; but if $\mu(q) \neq 0$, then $\sigma_{n}^{(a)}$ is a divergent sequence whose limit points constitute a circle with radius $|\mu(q)|$. Thus we obtain the following theorem which has several applications.

TheOREM 8.2. If $H(\chi)$ is regular and $R q=0$ but $q \neq 0$, then the sequence $s_{\boldsymbol{k}}^{(q)} \equiv k^{q}$ is summable $H(\chi)$ if and only if $\mu(q)=0$.

We now use Theorem 8.2 to prove Theorem 8.1. Let $H(\chi)$ be regular and let $q$ be a zero of $\mu(z)$ for which $R(q)=0$. Since regularity of $H(\chi)$ implies that $\mu(0)=1$, we have $q \neq 0$. Hence, by Theorem 8.2 , the sequence $k^{q}$ is summable $H(\chi)$. The sequence $k^{q}$ is the sequence of partial sums of a divergent series $\sum u_{n}$ for which $n\left|u_{n}\right|$ is bounded; hence an elementary Tauberian theorem implies that the sequence $k^{q}$ is not summable by the ordinary Abel method $A$. Since the series $\sum k^{q} z^{k}$ has radius of convergence 1 , it follows that the sequence $k^{q}$ is also not summable by the generalized Abel method $A^{*}$. Existence of the sequence summable $H(\chi)$ but not $A^{*}$ shows that $A^{*}$ does not include $H(\chi)$ and establishes Theorem 8.1.

Another consequence of Theorem 8.2 is set forth in the following theorem in which it is not assumed that the transformation $H\left(\chi_{1}\right)$ has an inverse.

THEOREM 8.3. If $H\left(\chi_{2}\right)$ and $H\left(\chi_{1}\right)$ are two regular Hausdorff methods such that $H\left(\chi_{2}\right)$ includes $H\left(\chi_{1}\right)$, then each zero of the moment function $\mu_{1}(z)$ of $\chi_{1}(t)$ with real part 0 is also a zero of the moment function $\mu_{2}(z)$ of $\chi_{2}(t)$. 
To prove this theorem, let $q$ be a zero of $\mu_{1}(z)$ for which $R q=0$. Then $q \neq 0$, and Theorem 8.2 implies that the sequence $k^{q}$ is summable $H\left(\chi_{1}\right)$. Hence $k^{q}$ is also summable $H\left(\chi_{2}\right)$ and Theorem 8.2 implies that $\mu_{2}(q)=0$.

In case $H\left(\chi_{1}\right)$ and $H\left(\chi_{2}\right)$ satisfy the hypothesis of Theorem 8.3 and $H\left(\chi_{1}\right)$ has an inverse $\left[H\left(\chi_{1}\right)\right]^{-1}$, then the transformation $H\left(\chi_{2}\right)\left[H\left(\chi_{1}\right)\right]^{-1}$ is a regular Hausdorff transformation $H\left(\chi_{3}\right)$ such that $H\left(\chi_{2}\right)=H\left(\chi_{3}\right) H\left(\chi_{1}\right)$, and the moment function $\mu_{3}(z)$ of $\chi_{3}(t)$ is such that

$$
\mu_{2}(z)=\mu_{3}(z) \mu_{1}(z),
$$$$
z \geqq 0 \text {. }
$$

This result was established by Hurwitz and Silverman [13] for the case in which $\mu_{1}(z)$ and $\mu_{2}(z)$ are analytic at $\infty$ and in a half-plane $R z>-\alpha$ for some $\alpha>0$; the extension to the more general regular transformations was made by Hille and Tamarkin [11] and by Garabedian, Hille, and Wall [7]. It thus appears that if $H\left(\chi_{1}\right)$ has an inverse, then the conclusion of Theorem 8.3 is a trivial consequence of (8.31). It would be interesting to know whether Theorem 8.3 could be strengthened by establishing existence of a regular moment function $\mu_{3}(z)$ satisfying (8.31) even when $H\left(\chi_{1}\right)$ does not have an inverse.

The sequences $k^{q}$, in which $R q=0, q \neq 0$, are not the only sequences of which the question of summability $H(\chi)$ is settled by vanishing or nonvanishing of a single moment of $\chi(t)$. Let $p$ be a positive integer and let

$$
s_{k}^{(p)}=k ! /(k-p) !, \quad k=0,1,2, \cdots,
$$

where $1 /(k-p)$ ! is interpreted to be 0 when $k=0,1, \cdots, p-1$. Let $H(\chi)$ be regular, and let $\sigma_{n}^{(p)}$ denote the $H(\chi)$ transform of $s_{n}^{(p)}$. Then

$$
\begin{aligned}
\sum_{k=0}^{n} C_{n, k} t^{k}(1-t)^{n-k} s_{k}^{(p)} & =\frac{n !}{(n-p) !} t^{p} \sum_{k=0}^{n-p} C_{n-p, k} t^{k}(1-t)^{n-p-k} \\
& =\frac{n !}{(n-p) !} t^{p}[t+1-t]^{n-p}=\frac{n !}{(n-p) !} t^{p}
\end{aligned}
$$

so that

$$
\sigma_{n}^{(p)}=\frac{n !}{(n-p) !} \int_{0}^{1} t^{p} d \chi(t)=\frac{n !}{(n-p) !} \mu(p) ;
$$

this formula was obtained by Silverman [19] by another method. It is a consequence of this formula that the sequence $n ! /(n-p) !$, in which $p$ is a positive integer, is summable by a regular Hausdorff method $H(\chi)$ if and only if $\mu(p)=0, \mu(z)$ being the moment function of $\chi(t)$. This implies that if $H\left(\chi_{2}\right)$ and $H\left(\chi_{1}\right)$ are regular Hausdorff methods with moment functions $\mu_{2}(z)$ and $\mu_{1}(z)$, and if $H\left(\chi_{2}\right)$ includes $H\left(\chi_{1}\right)$, then each positive integer zero of $\mu_{1}(z)$ must be a zero of $\mu_{2}(z)$. 
We are now in a position to prove the following theorem which, in particular, implies that the LeRoy method $L$ does not include $\mathfrak{H}$.

THEOREM 8.4. No totally regular method of summability can include $\mathfrak{F}$.

A method $T$ of summability is totally regular if it is regular, and if also the $T$ transform of each real sequence $s_{n}$ for which $s_{n} \rightarrow+\infty$ diverges to $+\infty$. Let $p$ be a positive integer and let $H(\chi)$ be a regular Hausdorff method such that the moment function $\mu(z)$ of $\chi(t)$ vanishes when $z=p$. Then the sequence $n ! /(n-p)$ ! is summable $H(\chi)$ to 0 . Hence the sequence is summable $\mathfrak{H C}$ to 0 . But when $T$ is totally regular, the sequence is not summable $T$. This proves Theorem 8.4.

\section{REFERENCES}

1. R. P. Agnew, The behavior of bounds and oscillations of sequences of functions under regular transformations, these Transactions, vol. 32 (1930), pp. 669-708.

2. - The effects of general regular transformations on oscillations of sequences of functions, these Transactions, vol. 33 (1931), pp. 411-424.

3. - Some remarks on a paper entitled "General Tauberian theorems," Journal of the London Mathematical Society, vol. 15 (1940), pp. 242-246.

4. E. Borel, Leçons sur les Fonctions Entières, Paris, 1900.

5. G. Doetsch, Über die Summabilität von Potenzreihen auf dem Rande des Borelschen Summabilitätspolygons, Mathematische Annalen, vol. 84 (1921), pp. 245-251.

6. H. L. Garabedian, On the relation between certain methods of summability, Annals of Mathematics, (2), vol. 32 (1931), pp. 83-106.

7. H. L. Garabedian, E. Hille, and H. S. Wall, Formulations of the Hausdorff inclusion problem, Duke Mathematical Journal, vol. 8 (1941), pp. 193-213.

8. H. L. Garabedian and H. S. Wall, Hausdorff methods of summation and continued fractions, these Transactions, vol. 48 (1940), pp. 185-207.

9. F. Hausdorff, Summationsmethoden und Momentfolgen, I and II, Mathematische Zeitschrift, vol. 9 (1921), pp. 74-109 and 280-299.

10. E. Hille, Essai d'une bibliographie de la representation analytique d'une fonction monogène, Acta Mathematica, vol. 52 (1929), pp. 1-80.

11. E. Hille and J. D. Tamarkin, Questions of relative inclusion in the domain of Hausdorff means, Proceedings of the National Academy of Sciences, vol. 19 (1933), pp. 573-577.

12. W. A. Hurwitz, Report on topics in the theory of divergent series, Bulletin of the American Mathematical Society, vol. 28 (1922), pp. 17-36.

13. W. A. Hurwitz and L. L. Silverman, On the consistency and equivalence of certain definitions of summability, these Transactions, vol. 18 (1917), pp. 1-20.

14. K. Knopp, Über das Eulersche Summierungsverfahren, I and II, Mathematische Zeitschrift, vol. 15 (1922), pp. 226-253 and vol. 16 (1923), pp. 125-156.

15. D. S. Morse, Relative inclusiveness of certain definitions of summability, American Journal of Mathematics, vol. 45 (1923), pp. 259-285.

16. E. Phragmén, Sur le domain de convergence de l'intégrale infinie $\int_{0}^{\infty} F(a x) e^{-a} d a$, Comptes Rendus de l'Académie des Sciences, Paris, vol. 132 (1901), pp. 1396-1399.

17. H. R. Pitt, General Tauberian theorems, Proceedings of the London Mathematical Society, (2), vol. 44 (1938), pp. 243-288.

18. H. Rademacher, Über den Konvergenzbereich der Eulerschen Reihentransformation, Sitzungsberichte der Berliner Mathematischen Gesellschaft, vol. 21 (1922), pp. 16-24. 
19. L. L. Silverman, On the omission of terms in certain summable series, Recueil Mathématique, vol. 33 (1926), pp. 375-384.

20. L. L. Silverman and J. D. Tamarkin, On the generalization of Abel's theorem for certain definitions of summability, Mathematische Zeitschrift, vol. 29 (1928), pp. 161-170.

Cornell University,

ITHACA, N. Y. 